\title{
Angiogenesis/Heparanase Inhibitor PG545
}

National Cancer Institute

\section{Source}

National Cancer Institute. Angiogenesis/Heparanase Inhibitor PG545. NCI Thesaurus.

Code C95202.

A synthetic heparan sulfate mimetic with potential anti-angiogenic and antineoplastic activity. PG545 inhibits the cleavage of heparan sulfate from cell surface proteoglycan by heparanase and thus inhibits the neovascularization induced by interaction between heparan sulfate and other extracellular matrix proteins. In this manner, this agent may have the potential to slow the progression of growth of solid tumors. 